\title{
Resposta fisiológica de sementes de palmeiras ao envelhecimento acelerado
}

\author{
Gisele de Freitas Negreiros ${ }^{(1)}$ e Sonia Cristina Juliano Gualtieri de Andrade Perez ${ }^{(1)}$
} (1)Universidade Federal de São Carlos, Dep. de Botânica, Caixa Postal 676, CEP 13565-905 São Carlos, SP. E-mail: gifn@terra.com.br,
dscp@power.ufscar.br

\begin{abstract}
Resumo - O objetivo deste trabalho foi avaliar a qualidade fisiológica de sementes de quatro espécies da família Palmae submetidas ao envelhecimento acelerado. Sementes recém-colhidas de cada uma das espécies foram expostas às condições de envelhecimento acelerado em câmara a $45^{\circ} \mathrm{C}$ e $100 \%$ de UR, durante os períodos de 0 (controle), 12, 24, 36 e 48 horas. Sementes de Euterpe edulis e Dypsis lutescens foram sensíveis ao tratamento, sendo a germinação totalmente suprimida após 12 horas de permanência na câmara. As espécies Roystonea oleracea e Phoenix reclinata apresentaram sementes mais resistentes que as outras espécies, mas redução gradativa do vigor, de acordo com o aumento do período de exposição às condições de envelhecimento; o período de 48 horas foi crítico para determinados parâmetros de vigor avaliados.
\end{abstract}

Termos para indexação: Palmae, longevidade de sementes, armazenamento de sementes, vigor, germinação de sementes.

\section{Physiological response of palm seeds to accelerated aging}

\begin{abstract}
The aim of this work was to evaluate the differences in seed physiological quality of four Palmae species submitted to accelerated aging. After harvest, the seeds of each species were exposed to accelerated aging $\left(45^{\circ} \mathrm{C}\right.$ and $100 \%$ R.U.) during 0 (control), 12, 24, 36 and 48 hours. Euterpe edulis and Dypsis lutescens were sensible to accelerated aging and germination process was inhibited after 12 hours inside the camara. Roystonea oleracea and Phoenix reclinata were more tolerant than the other species, but exibited gradual decrease on seed vigor, as the time inside the camara increased. The critical period for some vigor parameters evaluated was 48 hours inside the camara.
\end{abstract}

Index terms: Palmae, storage potential, seed germination, viability, vigor.

\section{Introdução}

A família Palmae ou Arecaceae compreende um grupo de plantas de grande importância econômica e ornamental, muito utilizadas em regiões tropicais, fornecendo ao homem, cocos, tâmaras, palmito, açúcar, sagu, óleo, cera, fibras e material para a construção de habitações rústicas, como folhas e estipe (Alves \& Demattê, 1987; Lorenzi et al., 1996).

A propagação das palmeiras se dá principalmente por sementes, que apresentam germinação lenta, irregular e freqüentemente em baixa porcentagem, para a maioria das espécies, perdendo a viabilidade rapidamente quando desidratadas (Broschat, 1994).
Um dos problemas relacionados ao armazenamento de espécies de palmeira $e x$-situ é a conservação de suas sementes, ou seja, como armazená-las por maior tempo sem perda da viabilidade. Sementes do gênero Euterpe, como Euterpe oleracea, Euterpe edulis, Euterpe espiritosantensis, são de difícil conservação por causa da sensibilidade à desidratação, sendo classificadas como recalcitrantes (Araújo et al., 1994; Andrade \& Pereira, 1997; Martins et al., 1999).

Na maioria das espécies de palmeiras, a viabilidade curta, durante o armazenamento, e a tolerância à desidratação diferem entre as espécies. A qualidade fisiológica de sementes de Phoenix loureiri durante o armazenamento foi severamente prejudicada quando o 
teor de umidade foi reduzido de $15,06 \%$ para $12,52 \%$ (Araújo \& Barbosa, 1992). Broschat \& Donselman (1987) consideraram que a perda de umidade em sementes recém-colhidas de Roystonea oleracea imediatamente semeadas pode ser maior que a ocorrida durante o armazenamento.

A longevidade da semente é uma característica da espécie e o armazenamento é importante para a conservação dos recursos genéticos em bancos de germoplasma, em que se busca a manutenção da viabilidade pelo maior tempo possível (Carneiro \& Aguiar, 1993).

O envelhecimento acelerado é um teste de vigor baseado na simulação de fatores ambientais adversos, temperatura e umidade relativa elevadas, que são relacionadas como causadoras da deterioração das sementes. Os processos de deterioração ocorridos neste teste são semelhantes aos que ocorrem no envelhecimento natural das sementes, porém, a uma velocidade acelerada (Delouche \& Baskin, 1973; Tekrony, 1993; Marcos Filho, 1994).

O objetivo deste trabalho foi avaliar e identificar diferenças na qualidade fisiológica das sementes de quatro espécies da família Palmae submetidas ao envelhecimento acelerado.

\section{Material e Métodos}

O trabalho foi realizado de 2000 a 2002 no laboratório de Ecofisiologia de Sementes e no jardim experimental do Departamento de Botânica da Universidade Federal de São Carlos, SP.

Foram utilizadas sementes recém-colhidas de quatro espécies de palmeiras. As sementes de Euterpe edulis Mart. foram provenientes do Estado de Santa Catarina, as de Roystonea oleracea (Jacq.) O.F. Cook foram fornecidas pelo Instituto de Pesquisa e Estudos Florestais (IPEF) em Piracicaba, SP, e as sementes de Dypsis lutescens $(\mathrm{H}$. Wendl) Beentje \& J. Dransf. e Phoenix reclinata Jacq. foram coletadas no Município de São Carlos, SP. Os frutos e sementes foram mantidos em sacos de polietileno durante o transporte entre o local de coleta e o beneficiamento no laboratório para assegurar o teor de umidade inicial.

Com exceção das sementes de Roystonea oleracea, que já foram recebidas beneficiadas, nas outras espécies, o trabalho de beneficiamento manual no laboratório constou da remoção do pericarpo e limpeza das sementes. Após a remoção da polpa dos frutos, as sementes foram lavadas em água corrente, seguida de lavagem em solução de hipoclorito de sódio a $2 \%$ durante 3 minutos e em água destilada. Em seguida, foram colocadas sobre papel toalha para secar à temperatura ambiente pelo período de dois a quatro dias, e selecionadas visualmente para garantir uniformidade de tamanho, coloração e estado de conservação. O material inerte e as sementes com sinais de ataque de insetos foram descartados.

$\mathrm{Na}$ determinação do teor de umidade, as sementes foram secadas em estufa à temperatura de $105^{\circ} \mathrm{C}$ durante 24 horas de acordo com as Regras para Análise de Sementes (Brasil, 1992), utilizando-se duas repetições de 25 sementes para cada espécie. Os resultados foram expressos em porcentagem de massa de água sobre massa da matéria fresca da semente. A seguir, os experimentos foram instalados mantendo-se o teor de umidade original de cada espécie recém-colhida.

No teste de envelhecimento acelerado, foram utilizadas amostras de 200 sementes para cada espécie, com quatro repetições de 50 sementes. Foram utilizadas caixas de plástico, onde uma prateleira de alumínio foi adaptada, e sobre a qual foram distribuídas as sementes, após a adição de $40 \mathrm{~mL}$ de água destilada (Marcos Filho, 1994). As caixas foram colocadas em câmara de envelhecimento acelerado à temperatura de $45^{\circ} \mathrm{C}$ e $100 \%$ de UR durante $0,12,24,36$ e 48 horas. A seguir, as sementes foram lavadas em solução de hipoclorito de sódio a $2 \%$ durante 3 minutos e em água destilada e submetidas ao teste de germinação em casa de vegetação com ventilação forçada e radiação solar de aproximadamente $72 \%$ (Andrade, 2001). Em caixas contendo $3 \mathrm{~kg}$ de areia grossa lavada e peneirada, foram semeadas quatro repetições de 50 sementes, à profundidade média de $2 \mathrm{~cm}$. A irrigação do substrato de areia foi realizada até a saturação, diariamente. As avaliações foram diárias, considerando-se como emergidas todas as sementes germinadas que emitiram a plúmula com $0,5 \mathrm{~mm}$ acima do substrato.

Foram avaliados os parâmetros de porcentagem de emergência, velocidade de emergência, velocidade de abertura foliar, tempo de abertura foliar após emergência, porcentagem de sobrevivência, tamanho médio e massa da matéria seca das plântulas .

Com os dados diários do número de plântulas normais, calculou-se a velocidade de emergência (Nakagawa, 1994) e, utilizando-se da mesma fórmula adaptada, calculou-se a velocidade de abertura foliar, computando-se o número de plântulas com folhas aber- 
tas da primeira à última contagem. A velocidade de abertura das folhas primárias foi calculada por contagem do número de dias após a semeadura. O tempo de abertura foliar foi calculado por contagem do número de dias após emergência da plúmula. $\mathrm{O}$ teste de germinação durou de 120 a 160 dias, de acordo com o comportamento germinativo característico de cada espécie. A taxa de sobrevivência foi expressa pela porcentagem de plantas vivas ao final do teste, em relação ao número total de plantas emergidas. As determinações do comprimento das plantas, da parte aérea e sistema radicular foram realizadas no último dia de avaliação, medindo-se a parte aérea do nível de inserção das sementes até a extremidade da folha, e a raiz, do mesmo ponto de inserção até a extremidade da maior raiz. A massa da matéria seca da parte aérea e radicular foi determinada mediante secagem a $80^{\circ} \mathrm{C}$ em estufa durante 48 horas ou até atingir peso constante.

O delineamento estatístico utilizado foi inteiramente casualizado, num esquema fatorial $4 \times 5$, ou seja, quatro espécies e cinco períodos de permanência na câmara de envelhecimento acelerado, com quatro repetições.
Os valores de porcentagem de germinação obtidos foram analisados pelo teste de Bartlett que não sugeriu transformação. Houve interação significativa entre os fatores para todas as avaliações e foram feitos os desdobramentos. Os dados foram submetidos à análise de variância e as médias comparadas pelo teste de Tukey a 5\% de probabilidade (Pimentel-Gomes, 2000).

\section{Resultados e Discussão}

O teor de umidade inicial das sementes de palmeiras determinado foi de 35\% em Dypsis lutescens, $37 \%$ em Euterpe edulis, $15 \%$ em Phoenix reclinata e 16\% em Roystonea oleracea.

A porcentagem de germinação inicial, sem exposição ao envelhecimento acelerado, foi significativamente diferenciada em três níveis, entre as quatro espécies da família Palmae estudadas (Tabela 1). Houve interação significativa entre as espécies e os períodos de envelhecimento. A espécie Phoenix reclinata foi a que apresentou maior porcentagem de germinação inicial, que foi reduzida com o aumento do período de exposição ao

Tabela 1. Efeito do envelhecimento acelerado na viabilidade e desenvolvimento inicial das plântulas de quatro espécies da família Palmae ${ }^{(1)}$.

\begin{tabular}{|c|c|c|c|c|c|c|c|c|c|c|c|}
\hline \multirow[t]{2}{*}{ Espécie } & \multirow[t]{2}{*}{$\mathrm{DAS}^{(2)}$} & \multicolumn{10}{|c|}{ Período de envelhecimento (horas) } \\
\hline & & Controle & 12 & 24 & 36 & 48 & Controle & 12 & 24 & 36 & 48 \\
\hline & & \multicolumn{5}{|c|}{ 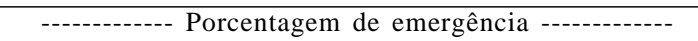 } & \multicolumn{5}{|c|}{ - Porcentagem de sobrevivência --------- } \\
\hline Dypsis lutescens & 120 & $53,0 \mathrm{Ab}$ & 0,0 & 0,0 & 0,0 & 0,0 & $91,09 \mathrm{Aa}$ & 0,00 & 0,00 & 0,00 & 0,00 \\
\hline Euterpe edulis & 159 & $44,0 \mathrm{Abc}$ & 0,0 & 0,0 & 0,0 & 0,0 & $92,06 \mathrm{Aa}$ & 0,00 & 0,00 & 0,00 & 0,00 \\
\hline Phoenix reclinata & 150 & $76,0 \mathrm{Aa}$ & $69,0 \mathrm{Aa}$ & $55,5 \mathrm{Ba}$ & $44,5 \mathrm{Ba}$ & $12,0 \mathrm{Ca}$ & $96,10 \mathrm{Aa}$ & $97,92 \mathrm{Aa}$ & $96,13 \mathrm{Aa}$ & $98,91 \mathrm{Aa}$ & $100,00 \mathrm{Aa}$ \\
\hline \multirow[t]{2}{*}{ Roystonea oleracea } & 116 & $37,0 \mathrm{Ac}$ & $44,0 \mathrm{Ab}$ & $42,0 \mathrm{Aa}$ & $33,0 \mathrm{Aa}$ & $8,0 \mathrm{Ba}$ & $83,83 \mathrm{Aa}$ & $100,00 \mathrm{Aa}$ & $98,86 \mathrm{Aa}$ & $100,00 \mathrm{Aa}$ & $71,43 \mathrm{Bb}$ \\
\hline & \multicolumn{6}{|c|}{-------- Velocidade de emergência ${ }^{(2)}$} & \multicolumn{5}{|c|}{ Velocidade da abertura foliar ${ }^{(2)}$} \\
\hline Dypsis lutescens & 120 & $69,74 \mathrm{Abc}$ & 0,00 & 0,00 & 0,00 & 0,00 & $104,69 \mathrm{Ab}$ & 0,00 & 0,00 & 0,00 & 0,00 \\
\hline Euterpe edulis & 159 & $85,33 \mathrm{Ab}$ & 0,00 & 0,00 & 0,00 & 0,00 & $127,16 \mathrm{Aa}$ & 0,00 & 0,00 & 0,00 & 0,00 \\
\hline Phoenix reclinata & 150 & $114,28 \mathrm{Aa}$ & $116,29 \mathrm{Aa}$ & $119,61 \mathrm{Aa}$ & $118,41 \mathrm{Aa}$ & $118,03 \mathrm{Aa}$ & $121,23 \mathrm{Aab}$ & $127,10 \mathrm{Aa}$ & $123,71 \mathrm{Aa}$ & $123,18 \mathrm{Aa} 1$ & $122,42 \mathrm{Aa}$ \\
\hline \multirow[t]{2}{*}{ Roystonea oleracea } & 116 & $63,33 \mathrm{Ac}$ & $63,31 \mathrm{Ab}$ & $56,72 \mathrm{ABb}$ & $59,36 \mathrm{Ab}$ & $43,73 \mathrm{Bb}$ & $79,51 \mathrm{Ac}$ & $79,90 \mathrm{Ab}$ & $75,13 \mathrm{Ab}$ & $76,25 \mathrm{Ab}$ & $52,76 \mathrm{Bb}$ \\
\hline & \multicolumn{6}{|c|}{ - } & \multicolumn{5}{|c|}{----- Porcentagem de plantas com folhas abertas ----- } \\
\hline Dypsis lutescens & 120 & $34,95 \mathrm{Aa}$ & 0,00 & 0,00 & 0,00 & 0,00 & $93,13 \mathrm{Aa}$ & 0,00 & 0,00 & 0,00 & 0,00 \\
\hline Euterpe edulis & 159 & $41,83 \mathrm{Aa}$ & 0,00 & 0,00 & 0,00 & 0,00 & $93,67 \mathrm{Aa}$ & 0,00 & 0,00 & 0,00 & 0,00 \\
\hline Phoenix reclinata & 150 & $8,06 \mathrm{Ac}$ & $10,81 \mathrm{Aa}$ & $6,67 \mathrm{Ab}$ & $4,78 \mathrm{Ab}$ & $10,91 \mathrm{Aa}$ & $94,21 \mathrm{Aa}$ & $89,37 \mathrm{Aa}$ & $81,05 \mathrm{Ab}$ & $89,71 \mathrm{Aa}$ & $84,37 \mathrm{Aa}$ \\
\hline \multirow[t]{2}{*}{ Roystonea oleracea } & 116 & $16,18 \mathrm{Ab}$ & $16,59 \mathrm{Aa}$ & $18,41 \mathrm{Aa}$ & $16,89 \mathrm{Aa}$ & $9,02 \mathrm{Ba}$ & $94,02 \mathrm{Aa}$ & $100,00 \mathrm{Aa}$ & $100,00 \mathrm{Aa}$ & $100,00 \mathrm{Aa}$ & $100,00 \mathrm{Aa}$ \\
\hline & & \multicolumn{5}{|c|}{------ Comprimento da parte aérea $(\mathrm{cm})$-.------ } & \multicolumn{5}{|c|}{------ Comprimento do sistema radicular $(\mathrm{cm})$------ } \\
\hline Dypsis lutescens & 120 & $9,45 \mathrm{Ab}$ & 0,00 & 0,00 & 0,00 & 0,00 & $6,91 \mathrm{Ab}$ & 0,00 & 0,00 & 0,00 & 0,00 \\
\hline Euterpe edulis & 159 & $11,51 \mathrm{Ab}$ & 0,00 & 0,00 & 0,00 & 0,00 & $11,25 \mathrm{Aa}$ & 0,00 & 0,00 & 0,00 & 0,00 \\
\hline Phoenix reclinata & 150 & $14,84 \mathrm{Aa}$ & $13,15 \mathrm{Ab}$ & $11,46 \mathrm{Ab}$ & $14,02 \mathrm{Ab}$ & $12,54 \mathrm{Aa}$ & 8,50Aab & 7,57Ab & 7,06Ab & 7,53Ab & $6,98 \mathrm{Ab}$ \\
\hline \multirow[t]{2}{*}{ Roystonea oleracea } & 116 & $18,60 \mathrm{Aa}$ & $20,35 \mathrm{Aa}$ & $22,67 \mathrm{Aa}$ & $20,80 \mathrm{Aa}$ & $17,66 \mathrm{Aa}$ & $11,72 \mathrm{Ba}$ & $13,58 \mathrm{Aa}$ & $15,85 \mathrm{Aa}$ & $13,21 \mathrm{Aa}$ & $10,75 \mathrm{Ba}$ \\
\hline & \multicolumn{6}{|c|}{--------- Matéria seca da parte aérea (g) --------- } & \multicolumn{5}{|c|}{------- Matéria seca do sistema radicular (g) -------- } \\
\hline Dypsis lutescens & 120 & $0,080 \mathrm{Ab}$ & 0,000 & 0,000 & 0,000 & 0,000 & $0,048 \mathrm{Ab}$ & 0,000 & 0,000 & 0,000 & 0,000 \\
\hline Euterpe edulis & 159 & $0,140 \mathrm{Aa}$ & 0,000 & 0,000 & 0,000 & 0,000 & $0,101 \mathrm{Aa}$ & 0,000 & 0,000 & 0,000 & 0,000 \\
\hline Phoenix reclinata & 150 & $0,096 \mathrm{Ab}$ & $0,087 \mathrm{Ab}$ & $0,079 \mathrm{Ab}$ & $0,095 \mathrm{Ab}$ & $0,093 \mathrm{Ab}$ & $0,045 \mathrm{Ab}$ & $0,049 \mathrm{Ab}$ & $0,048 \mathrm{Ab}$ & $0,046 \mathrm{Ab}$ & $0,041 \mathrm{Ab}$ \\
\hline Roystonea oleracea & 116 & $0,170 \mathrm{Aa}$ & $0,151 \mathrm{Aa}$ & $0,188 \mathrm{Aa}$ & $0,170 \mathrm{Aa}$ & $0,164 \mathrm{Aa}$ & $0,089 \mathrm{ABa}$ & $0,083 \mathrm{Ba}$ & $0,116 \mathrm{Aa}$ & $0,089 \mathrm{ABa}$ & $0,081 \mathrm{Ba}$ \\
\hline
\end{tabular}


envelhecimento. Roystonea oleracea manteve sua germinação até 36 horas de exposição ao envelhecimento acelerado, apresentando germinação significativamente inferior a Phoenix reclinata somente no controle $\mathrm{e}$ após 12 horas de envelhecimento acelerado. Dypsis lutescens e Euterpe edulis apresentaram grande sensibilidade aos efeitos adversos do envelhecimento e não germinaram a partir de 12 horas de permanência na câmara. Bovi \& Cardoso (1978) e Figliolia et al. (1987) identificaram a sensibilidade da espécie Euterpe edulis ao armazenamento e desidratação. Andrade et al. (1996) observaram que as sementes de Euterpe edulis, armazenadas com $37 \%$ de umidade, apresentaram bons resultados de germinação; observaram ainda altos índices de velocidade de emergência aos dois meses de armazenamento nas sementes armazenadas com $37 \%$, $40 \%$ e $43 \%$ de umidade.

Quanto à sobrevivência, não houve diferença significativa entre as espécies na situação controle. Phoenix reclinata e Roystonea oleracea tiveram boa sobrevivência após o envelhecimento, sendo significativa a diminuição da sobrevivência na espécie Roystonea oleracea, apenas no período de envelhecimento de 48 horas (Tabela 1 ).

A condução do experimento em casa de vegetação variou em média de quatro a cinco meses, e foi concluído quando cada espécie apresentou, no mínimo, 90\% das plantas vivas com folhas abertas (Tabela 1). O envelhecimento acelerado não acarretou mudanças significativas nas duas espécies resistentes ao envelhecimento, exceto no período de 24 horas, em que Phoenix reclinata apresentou porcentagem de plantas com folhas abertas inferior a Roystonea oleraceae .

A velocidade de emergência nas sementes das quatro espécies não envelhecidas diferiu significativamente, e a espécie Phoenix reclinata precisou de maior número de dias para a emergência, enquanto Roystonea oleracea foi a espécie que emergiu mais rapidamente e Euterpe edulis e Dypsis lutescens apresentaram um comportamento intermediário (Tabela 1). Observou-se que, nos períodos de 12 a 48 horas de envelhecimento, a espécie Roystonea oleracea emergiu mais rapidamente que Phoenix reclinata. Broschat \& Donselman (1987) observaram que sementes de Roystonea oleracea armazenadas em sacos de polietileno fechados à temperatura de $23^{\circ} \mathrm{C}$ mantiveram a viabilidade até nove meses, e germinaram melhor que as sementes recém-colhidas. Maciel (2001), estudando Roystonea oleracea, observou que não houve diferença entre a velocidade de emergência de sementes recém-colhidas e sementes armazenadas em sacos de polietileno por 12 semanas à temperatura ambiente. Porém houve redução da porcentagem de emergência, e este autor sugere que pode haver dormência em sementes armazenadas de Roystonea oleracea. Os resultados de porcentagem e velocidade de emergência para Roystonea oleracea após o envelhecimento acelerado foram superiores aos obtidos por Maciel (2001) em observação durante 35 semanas. $\mathrm{O}$ aumento dos valores de porcentagem de emergência após envelhecimento acelerado comparado com o controle poderia ser explicado pela hipótese de que as sementes sofreram, durante alguns dos períodos de permanência na câmara, hidratação suficiente para iniciar o processo germinativo.

Não houve efeitos significativos na velocidade de emergência na espécie Phoenix reclinata nos períodos de 12 a 48 horas de envelhecimento acelerado. $\mathrm{Na}$ Roystonea oleracea, após 48 horas de envelhecimento, houve redução significativa dos dias exigidos para emergência das plântulas.

A velocidade de abertura foliar nas quatro espécies de sementes não envelhecidas diferiu significativamente. Roystonea oleracea foi a palmeira com abertura foliar mais rápida, seguida por Dypsis lutescens e, por último, as espécies Phoenix reclinata e Euterpe edulis (Tabela 1). Não houve efeito dos períodos de envelhecimento sobre a velocidade de abertura foliar na espécie Phoenix reclinata. As plântulas de Roystonea oleracea mantiveram a velocidade de abertura foliar semelhante ao controle, até o período de 36 horas de envelhecimento. No período de 48 horas, as sementes de Roystonea oleracea apresentaram redução significativa no tempo médio de abertura foliar. Comparandose as espécies Phoenix reclinata e Roystonea oleracea nos períodos de envelhecimento de 12 a 48 horas, observou-se efeito semelhante ao causado à velocidade de emergência, sendo novamente Roystonea oleracea a espécie que levou menor número de dias após a semeadura para a abertura das folhas (Tabela 1$)$.

Quanto ao tempo entre a emergência da plúmula e a abertura foliar, observou-se que a espécie que apresentou menor tempo foi Phoenix reclinata, seguida respectivamente das espécies Roystonea oleracea, Dypsis lutescens e Euterpe edulis (Tabela 1).

Em relação ao tamanho das plântulas emergidas das sementes não envelhecidas, o tamanho médio da parte 
aérea das espécies Phoenix reclinata e Roystonea oleracea foi significativamente superior às espécies Dypsis lutescens e Euterpe edulis (Tabela 1). O envelhecimento acelerado não apresentou efeito significativo no tamanho da parte aérea das espécies Phoenix reclinata e Roystonea oleracea, mas houve diferenças entre elas nos períodos de 12 a 36 horas, e a espécie Roystonea oleracea foi superior nestes períodos, apresentando maior comprimento da parte aérea (Tabela 1). As espécies Roystonea oleracea e Euterpe edulis apresentaram maior comprimento da maior raiz. Phoenix reclinata apresentou tamanho radicular intermediário e Dypsis lutescens exibiu o menor comprimento do sistema radicular. Não houve efeito do envelhecimento acelerado no comprimento do sistema radicular de Phoenix reclinata em todos os períodos de envelhecimento. Entre as espécies Roystonea oleraceae e Phoenix reclinata, a primeira apresentou tamanho do sistema radicular maior em todos os períodos de envelhecimento (Tabela 1 ). Na espécie Roystonea oleracea, os períodos de envelhecimento de 12, 24 e 36 horas proporcionaram um incremento significativo no tamanho do sistema radicular, em comparação com o grupo controle.

Não houve efeito do envelhecimento acelerado sobre a massa da matéria seca da parte aérea das plantas dentro de cada espécie avaliada, mas houve diferenças entre as espécies no grupo controle e nos períodos de envelhecimento (Tabela 1). A massa da matéria seca da parte aérea das sementes não envelhecidas foi maior em Roystonea oleracea e Euterpe edulis e menor em Phoenix reclinata e Dypsis lutescens. Nos períodos de 0 até 48 horas de envelhecimento, Roystonea oleracea produziu maior quantidade de matéria seca que Phoenix reclinata.

Houve diferença significativa entre as espécies no grupo controle e nos períodos de envelhecimento, com relação à massa da matéria seca do sistema radicular, efeito similar ao ocorrido com a avaliação da massa da matéria seca da parte aérea. Na espécie Roystonea oleracea, o período de 24 horas de envelhecimento acelerado proporcionou aumento da massa do sistema radicular, e houve redução nos períodos de 12 e 48 horas (Tabela 1).

Fatores relacionados intrinsecamente com as sementes, como diferenças entre espécies, nível inicial de vigor de cada lote, teor de umidade inicial, condições da planta-mãe e do local de produção das sementes foram naturalmente diferenciados entre as quatro espécies.
A compreensão destas diferenças iniciais permitiu melhor comparação entre as quatro espécies submetidas ao envelhecimento acelerado, com posterior avaliação do desenvolvimento em casa de vegetação.

\section{Conclusões}

1. As espécies Dypsis lutescens e Euterpe edulis apresentam maior sensibilidade aos efeitos adversos do envelhecimento acelerado.

2. O período de 48 horas de envelhecimento acelerado é crítico para vários parâmetros de vigor avaliados nas espécies Phoenix reclinata e Roystonea oleracea.

\section{Referências}

ALVES, M.R.P.; DEMATTÊ, M.E.S.P. Palmeiras: características botânicas e evolução. Campinas: Fundação Cargill, 1987. 129p.

ANDRADE, A.C.S. de. Influência da saturação hídrica do solo e do sombreamento no crescimento de plântulas jovens de matas ribeirinhas. 2001, 139p. Tese (Doutorado) - Universidade Federal de São Carlos, São Carlos.

ANDRADE, A.C.S. de; MALAVASI, M.M.; COSTA, F.A. da. Conservação de palmiteiro (Euterpe edulis Mart.): efeito da temperatura de armazenamento e do grau de umidade das sementes. Revista Brasileira de Sementes, v.18, p.149-155, 1996.

ANDRADE, A.C.S. de; PEREIRA, T.S. Comportamento de armazenamento de sementes de palmiteiro (Euterpe edulis Mart.). Pesquisa Agropecuária Brasileira, v.32, p.987-991, 1997.

ARAÚJO, E.F.; BARBOSA, J.G. Influência da embalagem e do ambiente de armazenamento na conservação de sementes de palmeira (Phoenix loureiri Kunth). Revista Brasileira de Sementes, v.14, p.61-64, 1992.

ARAÚJO, E.F.; SILVA, R.F. da; ARAÚJO, R.F. Avaliação da qualidade de sementes de açaí armazenadas em diferentes embalagens e ambientes. Revista Brasileira de Sementes, v.16, p.76-79, 1994.

BOVI, M.L.A.; CARDOSO, M. Coservação de sementes de palmiteiro (Euterpe edulis Mart.). Bragantia, v.37, p.67-71, 1978.

BRASIL. Regras para análise de sementes. Brasília: Ministério da Agricultura. Departamento Nacional de Produção Vegetal, 1992. $365 \mathrm{p}$.

BROSCHAT, T.K. Palm seed propagation. Acta Horticulturae, n.360, p.141-147, 1994.

BROSCHAT, T.K.; DONSELMAN, H. Effects of fruit maturity, storage, presoaking, and seed cleaning on germination in three species of palms. Journal of Enviromental Horticulture, v.5, p.6-9, 1987.

CARNEIRO, J.G. de A.; AGUIAR, I.B. Armazenamento de sementes. In: AGUIAR, I.B. de; PIÑA-RODRIGUES, F.C.M.; FIGLIOLIA, M.B. (Coord.). Sementes florestais tropicais. Brasília: Abrates, 1993. p.333-350. 
DELOUCHE, J.C.; BASKIN, C.C. Accelerated aging techniques for predicting the relative storability of seed lots. Seed Science and Technology, v.1, p.427-452, 1973.

FIGLIOLIA, M.B.; SILVA, A.; YAMAZOE, G.; SIQUEIRA, A.C.M.F. Conservação de sementes de Euterpe edulis Mart. em diferentes embalagens e ambientes de armazenamento. Boletim Técnico do Instituto Florestal, v.41, p.355-368, 1987.

LORENZI, H.; SOUZA, H.M. de; MEDEIROS-COSTA, J.T. de; CERQUEIRA, L.S.C. de; BEHR, N. von. Palmeiras no Brasil: nativas e exóticas. Nova Odessa: Plantarum, 1996. 303p.

MACIEL, N. Emergência de la palma real venezoelana (Rosytonea oleraceae (Jacq.) O.F. Cook) em función de condiciones variables del fruto y la semilla. Bioagro, v.13, p.105-110, 2001.
MARCOS FILHO, J. Teste de envelhecimento acelerado. In: VIEIRA, R.D.; CARVALHO, N.M. (Ed.). Testes de vigor em sementes. Jaboticabal: Funep, 1994. p.133-150.

MARTINS, C.C.; NAKAGAWA, J.; BOVI, M.A.L. Tolerância à dessecação de sementes de palmito-vermelho (Euterpe espiritosantensis Fernandes). Revista Brasileira de Botânica, v.22, p.391-396, 1999.

NAKAGAWA, J.T. Testes de vigor baseados na avaliação de plântulas. In: VIEIRA, R.D.; CARVALHO, N.M. (Ed.). Testes de vigor em sementes. Jaboticabal: Funep, 1994. p.49-85.

PIMENTEL-GOMES, F. Curso de Estatística experimental. 14.ed. Piracicaba: Esalq, 2000. 477p.

TEKRONY, D.M. Accelerated aging test. Journal of Seed Technology, v.17, p.110-120, 1993. 